\title{
Modeling the motion processes of a multifunctional robot for animal units
}

\author{
Evgenii Nikitin ${ }^{1, *}$, and Dmitrii Pavkin ${ }^{1}$ \\ ${ }^{1}$ Federal Scientific Agroengineering Center VIM, 1st Institute pas. 5, Moscow, 109428, Russia
}

\begin{abstract}
The goal of the research is a mathematical modeling of the motion process of a wheeled robot along a given trajectory with the turn drill. Matlab software with the Simulink option package was used as the environment for artificial modeling of the motion process. In order to control the movement of a wheeled robot using the computational and graphic methods, a kinematic and dynamic model of a wheeled robot was determined. The robot has two independent drive wheels located on the same axis and operating on electric traction. Based on the obtained mathematical correlations, a nomenclature of artificial components was formed in the Matlab (Simulink) environment, which made possible to simulate the process of controlling the robot's electric drive. A voltage that was twice as different was applied to the electric drive of independent wheels located on the same axis as a reliability check of the mathematical model in the Matlab (Simulink) artificial medium. The artificially simulated motion process of a wheeled robot involved studying the motion process using the "PLOT (X; Y)" function. At the moment of the robot's movement, the trajectory of the $\boldsymbol{O}_{\mathbf{0}}$ point, which is the center of robot's mass, for a set unit of time, was obtained. This allowed confirming the reliability of the developed mathematical model for the control system of a multifunctional robot for animal units.
\end{abstract}

\section{Introduction}

The process of keeping cattle in animal units involves the implementation of many cyclical, labor-intensive operations, while forming various indicators of technological production efficiency, determined by labor productivity. This becomes a determinant factor for designing the technological equipment, so most manufacturers strive to automate or robotize the various operations performed by humans.

Some of the most labor-intensive operations in the cattle keeping are the processes of feeding farm animals and manure removal. The first most strongly affects the level of animal productivity, which is the main indicator characterizing the capital productivity of production. The second has a significant impact on the microclimate in the building of the animal unit, and also is responsible for the hygiene of the room.

\footnotetext{
${ }^{*}$ Corresponding author: evgeniy.nicks@yandex.ru
} 
According to the previous studies, the main problem of technical and technological support of the feeding quality at animal units for cattle milk production is the accuracy of dosing and the efficiency of mixing concentrated high-energy feed components [1].

As an alternative solution to oft-stated problem, the designing of a robotic platform is proposed, which will increase the productivity of labor and the efficiency of performing heavy difficult operations for the maintenance of cattle in animal units. The attached pieces for autonomous servicing of the manger can be used as off-chip devices for a robotic platform. They can also be used for distribution of feed, dosing of concentrated additives that enhance the taste of the main feed, cleaning of technological surfaces, partial manure removal.

Parray, Rouf and others presented various results of research on agricultural machinery showing the wide potential for robotization of the designed machines on the example of a rice harvester. [2].

It is necessary to take into account a number of kinematic and geometric parameters of robot junctions under various scenarios when creating a multi-functional wheeled robot for autonomous execution of various technological operations, as was indicated in the studies of Subhashree, S.N., Igathinathane, C. [3]. It is also necessary to take into account the results of a research by Crolla, D.A., \& El-Razaz, A.S.A., which allow considering the effect of combined lateral and longitudinal forces created by tires on deformable surfaces [4], when modeling the motion process of a robotic vehicle. These forces must be taken into account when drilling the rotation of the developed wheeled robot on rubber mats mounted on the surface of the serviced space inside the animal unit. According to the studies of Mileiko, S., Bunnam, T., Xia, F., Shafik, R., Yakovlev, A., \& Das, S. [5], it was found that the creation of artificial neural systems for applications of artificial intelligence requires considering physical parameters of a controlled object [5], in the role of which a wheeled robot acts as part of our research.

The idea of developing a multifunctional robotic platform for performing various technological operations in animal units was born through a detailed analysis of development trends of modern robotics for agriculture, such manufacturers known in the world market as Lely Group - Netherlands, Delaval - Sweden, Wasserbauer - Austria, Gea Farm Technology - Germany and others. One of the most popular areas in the development of robotic machines is wheeled robotic platforms facilitating technological processes of preparing the feed mixture, distributing it, as well as the process of manure removal. The relevance of creating robotic autonomous controlled systems with electric drive can be traced in other areas of consumption, as was described in studies by Chen, Q. et al. [6].

It is assumed that the developed robotic platform will be controlled by using an intelligent system and sensors of the Internet of Things, similarly described in studies by Mahalakshmi, J., Kuppusamy, K., Kaleeswari, C., \& Maheswari, P. [7].

Also, based on the results of the study by He, P., \& Li, J, the robot control system will develop a routing and a report on the technological impacts made [8]. It is planned to use a nonlinear vehicle stability control system as a platform stability control [9].

Also, additional requirements on the quality of positioning are imposed when creating a robotic platform, the autonomous movement of which will be carried out inside industrial buildings. Therefore, the experience of Barawid, O.S. et al. in designing an autonomous navigation system using a two-dimensional laser scanner was studied in detail. The use of such navigation system allows elimiating the use of GPS and GLANAS systems, which do not provide the required accuracy of the positioning of the developed machine inside various buildings [10].

Therefore, it is necessary to develop a mathematical description of the movement of the wheeled robotic platform throughout the process of creating a unified robotic platform for 
performing maintenance operations. This will provide the most accurate digital connection between the positioning system and the movement of the wheeled robot along the plane.

Let us carry out the process of modeling the movement of the developed robot with a simulation of turning, while forming a circle graph that describes the mass center trajectory of the robot during movement.

\section{Goal of research}

The aim of the presented research is a mathematical simulation of the motion process of a wheeled robot along a given trajectory with the turning drill.

\section{Materials and methods}

The mathematical modeling method was applied for carrying out the study, and the Matlab/Simulink software package was used.

\subsection{Simulating the drive systems of wheeled robot}

In order to describe the kinematics of a small-sized wheeled robot, the process of moving along a plane was considered, as well as moving along a rigidly defined trajectory, which is shown in figure 1.

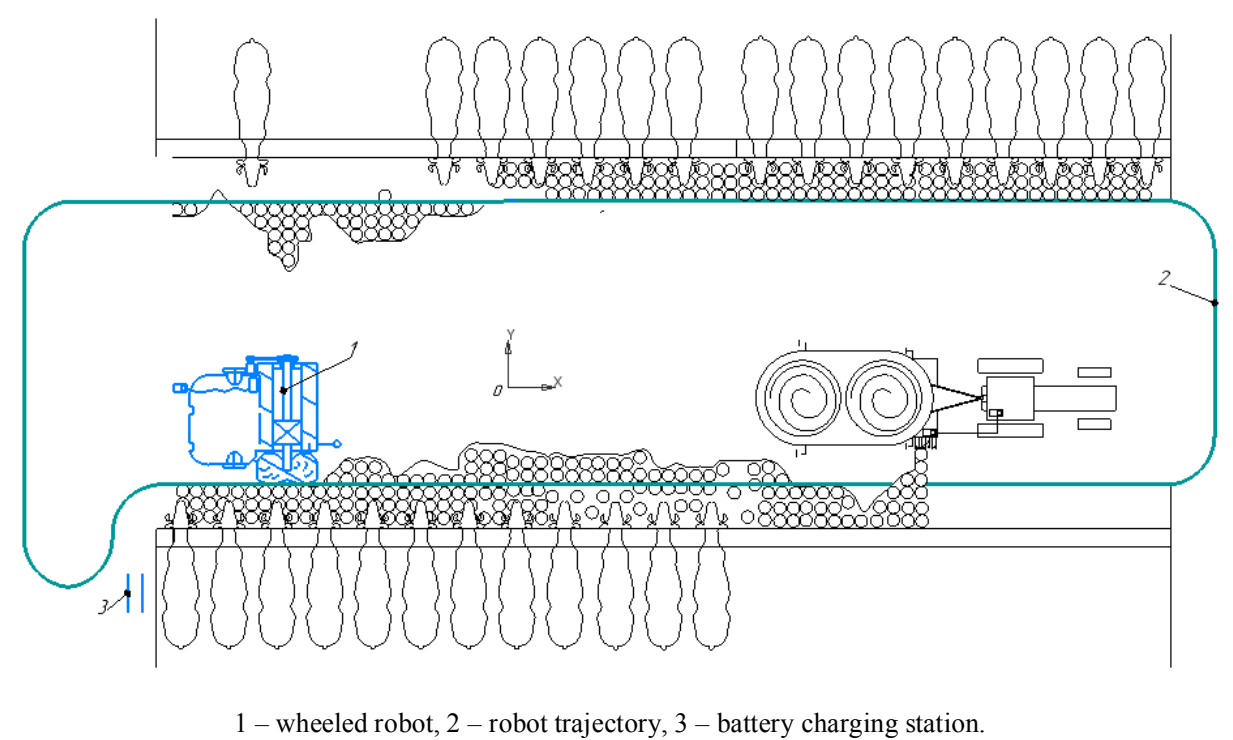

Fig. 1. Schematic diagram of the robot movement in the animal unit.

Thus, the plane motion of the robot in figure 1 is in the $X o Y$ plane, with the center of mass of the robot $O_{0}$, associated with the moving coordinate system $X_{0} O_{0} Y_{0}$

The simulation process assumes that the wheels are independently controlled and rotate without slipping, the center distance of the wheels is $O_{0} O_{1}=O_{0} O_{2}=a$.

The position of each wheel is characterized by the Cartesian coordinates of the $K_{i}$ point, the course angle $\varphi$ and the rotation angle of the wheel $\Omega_{i}$, and is presented in the design diagram in figure 2 . 

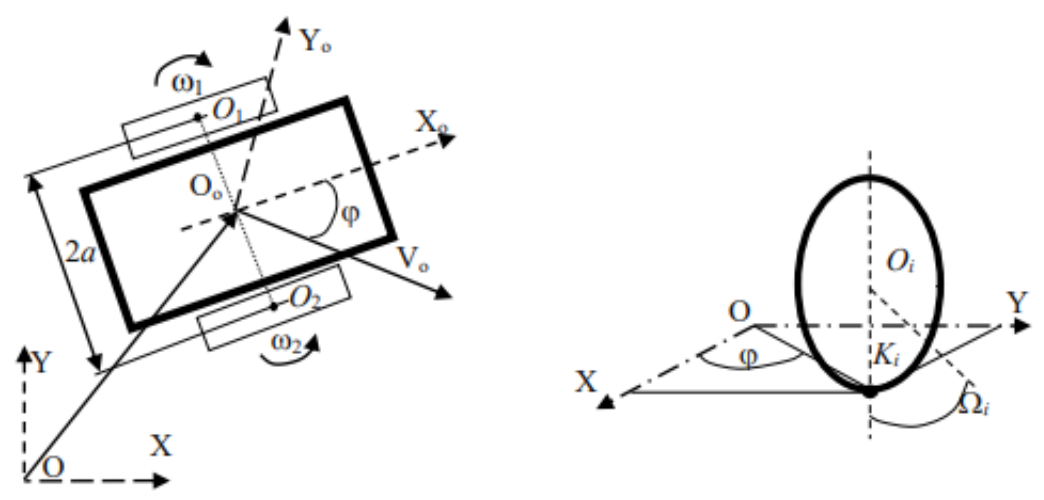

Fig. 2. Analytical model representing the kinematics of the robot's movement.

The motion process of wheels is followed by systems of equations $(1,2)$ :

$$
\begin{gathered}
\left\{\begin{array}{l}
\dot{x_{1}}=0.5 \mathrm{~d} \times \omega_{1} \times \operatorname{Cos} \varphi \\
\dot{y_{1}}=0.5 \mathrm{~d} \times \omega_{1} \times \operatorname{Sin} \varphi
\end{array}\right. \\
\left\{\begin{array}{l}
\dot{x_{2}}=0.5 d \times \omega_{2} \times \operatorname{Cos} \varphi \\
\dot{y_{2}}=0.5 d \times \omega_{2} \times \operatorname{Sin} \varphi
\end{array}\right.
\end{gathered}
$$

where

$x_{1}, x_{2}, y_{1}, y_{2},-$ coordinates of points $K_{1}$ and $K_{2}$;

$\mathrm{d}$ - wheel diameter;

$\omega_{1}=\Omega_{1}-$ angular velocity of the wheel 1 ;

$\omega_{2}=\Omega_{2}-$ angular velocity of the wheel 2 ;

$$
V_{0}=0.25 d \times\left(\omega_{1}-\omega_{2}\right), \operatorname{tg} \varphi=\frac{V_{0}}{R+a}=\frac{0.25 d \times\left(\omega_{1}-\omega_{2}\right)}{R+a}
$$

where

$\mathrm{R}=\mathrm{O}_{0} \mathrm{O}_{2}$ - turning radius of the robot;

$\mathrm{a}$ - design parameter of the model.

The geometric coordinates of the mass center of the robot is the middle of the $O_{1} O_{2}$ segment, which is the center of the wheelspace followed by the system of equations (4)

$$
\left\{\begin{array}{l}
x_{0}=\frac{x_{1}+x_{2}}{2} \\
y_{0}=\frac{y_{1}+y_{2}}{2}
\end{array}\right.
$$

The geometric constraint is followed by the system of equations (5)

$$
\left\{\begin{array}{l}
x_{2}=2 a \times \operatorname{Cos} \varphi+x_{1} \\
y_{2}=2 a \times \operatorname{Sin} \varphi+y_{1}
\end{array}\right.
$$

The set of equations (1-5) is a kinematic model of the movement of the robot.

The description of the dynamic motion process model of the robot was carried out using the Newton-Euler method. 


$$
\left\{\begin{array}{l}
m \ddot{x_{0}}=\sum F_{x} \\
m \ddot{y_{0}}=\sum F_{y} \\
J_{0} \ddot{\varphi}=\sum M_{0}
\end{array}\right.
$$

where

m - robot's mass;

$J_{0}$ - inertia tensor of the transport robot relative to the associated coordinate system $X_{0} O_{0} Y_{0}$.

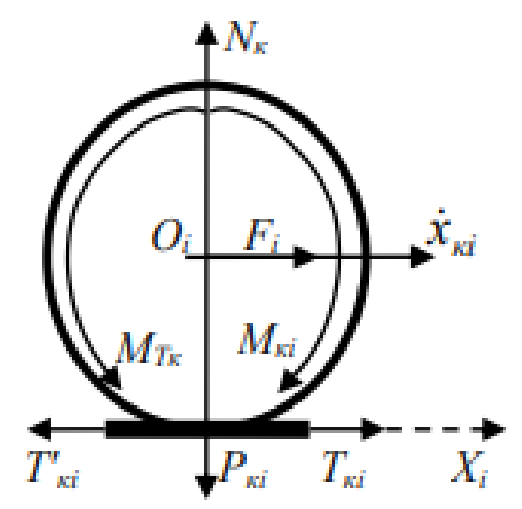

Fig. 3. Analytic model representing the robot's dynamics.

Projection of forces $F_{1}$ and $F_{2}$ on the axis $\mathrm{X}$

$$
\sum F_{x}=0.5\left(F_{1 x}+F_{2 x}\right)
$$

Projection of forces $F_{1}$ and $F_{2}$ on the axis $\mathrm{X}$

$$
\sum F_{y}=0.5\left(F_{1 y}+F_{2 y}\right)
$$

The moment of forces $F_{1}$ and $F_{2}$ relative to the robot's center of mass.

$$
\sum M_{0}=a\left(F_{1}-F_{2}\right)
$$

According to figure 3, which is an analytic model representing the motion dynamics of a wheeled robot, the movement of a separately-loaded wheel was considered.

It was assumed that a torque $M_{k i}$ was applied to the drive wheel, as well as the external moment $M_{i}$ and the frictional torque $M_{T k i}$ (fig. 3). The external forces with respect to the wheel are the following: wheel weight $P_{k i}$, normal road reaction $N_{k i}$, external load force $F_{i}$ and friction force $T_{k i}$. The case when $N_{k i} \geq P_{k i}$ is considered.

A differential equation for the wheel motion was compiled, provided that $N_{k i}=N_{k i}$; $Y_{k i}=0$. 


$$
\left\{\begin{array}{c}
m_{k i} \ddot{x_{k l}}=F_{i}-T_{k i}^{\prime}+T_{k i} \\
J_{\sum i} \times \omega_{l}=\left(T_{k l}-T_{k l}^{\prime}\right) \times r_{k}-M_{\sum i} \\
M_{\sum i}=M_{k i}-k \times N_{k i}
\end{array}\right.
$$

where,

$r_{k}=0.5 d-$ wheel radius;

$\mathrm{k}$ - coefficient of rolling resistance,

$\omega_{i}$ - angular velocity of the wheel,

$m_{k i}-$ wheel mass,

$J_{\sum i}$ - total inertia moment of the wheel,

$\mathrm{M}_{T k i}=\mathrm{k} \times N_{k i}$

The moment $M_{k i}$ created by an electric drive that rotates the wheel through the gearbox with the gear ratio $\eta(\eta<1)$,

$$
\left\{\begin{array}{c}
\omega_{g}=\eta \times \omega_{i} \\
J \sum i=J_{k i}+\widetilde{J_{g \imath}}
\end{array}\right.
$$

where,

$\omega_{g}$ - rotor angular velocity;

$J_{k i}=\frac{m_{k i} \times r_{k}^{2}}{2}-$ inertia moment of the wheel;

$J_{g i}=\frac{J_{g i}}{\eta^{2}}-$ the inertia moment of the rotor of the electric motor, reduced to the axis of the wheel.

If the wheel rolls without sliding, the point of contact of the wheel with the road $K_{i}$ is the instantaneous center of speed, and $\overline{x_{k l}}$ is the speed when rotating around the instantaneous center of speed,

$$
\begin{gathered}
\left\{\begin{array}{l}
T_{k i}=-r_{k} \times \omega_{i} \\
x_{k i}=-r_{k} \times \dot{\omega}_{l}
\end{array}\right. \\
T_{k i}-T_{k i}^{\prime}=\frac{m_{k i} \times r_{k} \times M_{\sum i}-J_{\sum i} \times F_{i}}{m_{k i} \times r_{k}^{2}+J_{\sum i}} \\
\ddot{x_{k l}}=\frac{F_{i}+T_{k i}^{\prime}-T_{k i}}{m_{k i}}=\frac{r_{k}\left(M_{\sum i}+F_{i} \times r_{k}\right)}{m_{k i} \times r_{k}^{2}+J_{\sum i}}
\end{gathered}
$$

The angular velocity value of the wheel $\omega_{i}$ is determined by the integration of equations (10) or (13).

When the wheels move without slipping, the condition $T_{k i} \leq \cdot f$ should be met, where $f$ is the friction coefficient between the wheel and the road; the wheel will slip in case when $T_{k i} \geq \cdot f$.

Force $F_{i}$ can be considered as a reaction of the robot model to the i-th wheel

$$
F_{i}=T_{c p i}-T_{k i}
$$


where,

$T_{c p i}$ - average driving force of the wheel, determined by using the following the formula (16)

$$
T_{c p i}=\sum_{i=1}^{2} \frac{T_{k i}}{2}
$$

In case when the design parameters of the model are known, the forces can be determined by using formulas (13-15), as well as the moments acting on each wheel. Thus, the right-hand sides of the system of equations (5) are calculated. The set of equations (7-9, $13,14)$ is a dynamic model of a robot with a drive in the form of two independent wheels.

\subsection{Analytical model of the robot}

The motor stator circuit equation is represented by the formula (16)

$$
L_{S T}=\frac{d I_{S T}}{d t}+R_{S T} \times I_{S T}+C_{\Sigma} \times \omega_{g}=U_{g}
$$

where,

$L_{S T}$ - stator winding inductance,

$R_{S T}$ - stator winding resistance,

$I_{S T}$ - stator winding current,

$\omega_{g}$ - rotor angular velocity,

$U_{g}$ - control voltage of stator winding.

The engine torque is followed by equation (17)

$$
M_{g}=C_{M} \times J_{S T}
$$

The equation of torque for the wheel is determined by formula (18)

$$
M_{k i}=\frac{\mu}{\eta} \times M_{g}
$$

$\mu$-gearbox efficiency, $\eta-$ reduction coefficient $(\eta<1)$.

The driving force of the wheel is determined by the following equation (19)

$$
T_{k i}=\frac{m_{k} \times r_{k} \times M_{\sum i}}{m_{k} \times r_{k}^{2}-2 \times J_{\sum i}}
$$

The acceleration of the robot is determined by the following equation (20)

$$
\ddot{x_{m}}=\frac{\sum T_{k i}}{m_{m}}
$$

The linear speed of the wheel is followed by equation (21)

$$
x_{\dot{m} k l}^{\cdot}=\int x_{m k l}^{\ddot{~}} d t
$$

The angular velocity of the wheel is given by equation (22) 


$$
\omega_{i}=-\frac{x_{\mathrm{m} k l}^{\cdot}}{r_{k}}
$$

The angular velocity of the motor output shaft is followed by equation (23)

$$
\omega_{g}=\frac{\omega_{i}}{r}
$$

The feedback equation (24)

$$
U_{f b}=K_{f b} \times \omega_{f}
$$

where,

$U_{f b}$ - feedback voltage;

$K_{f b}$ - feedback coefficient;

$\omega_{\int}-$ actual value of angular velocity.

\subsection{Simulating the mathematical model of the robot in the programming environment Matlab/Simulink}

The element base with two independent drive wheels was modeled in a Matlab/Simulink environment as a mathematical model simulation of a wheeled robot (figure 4).

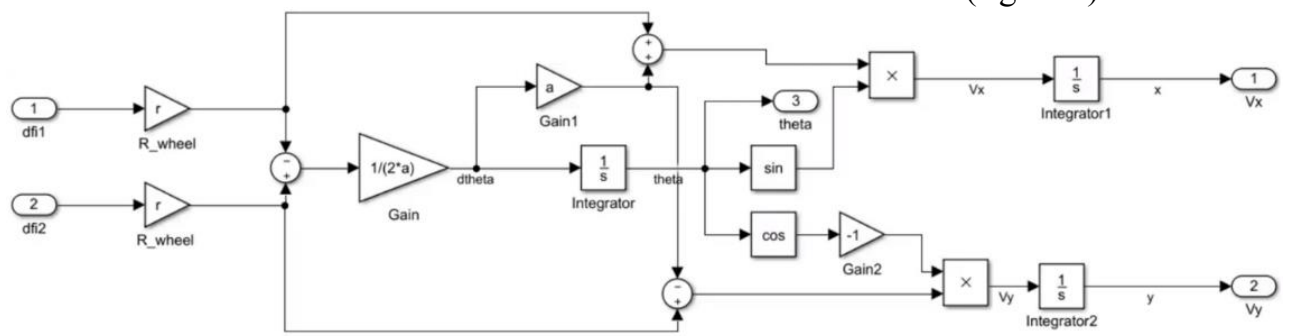

Fig. 4. Elements of simulating the wheeled robot's drive in a Matlab/Simulink environment.

The result of simulating the mathematical model of a wheeled robot with two independent drive wheels is the construction of a graph in the Matlab/Simulink environment, which is obtained by describing the mass center of the robot in the (x,y) plane. Moreover, indicators Constant $=5$, Constant $1=10$, which characterizes the level of voltage supply to the wheel drive.

Thus, the mathematical model of the robot's motion can be considered reliable, because the trajectory obtained by using the PLOT (x;y) function in the Matlab/Simulink environment is a circle (figure 6), due to the difference in the applied voltage to the wheel drives.

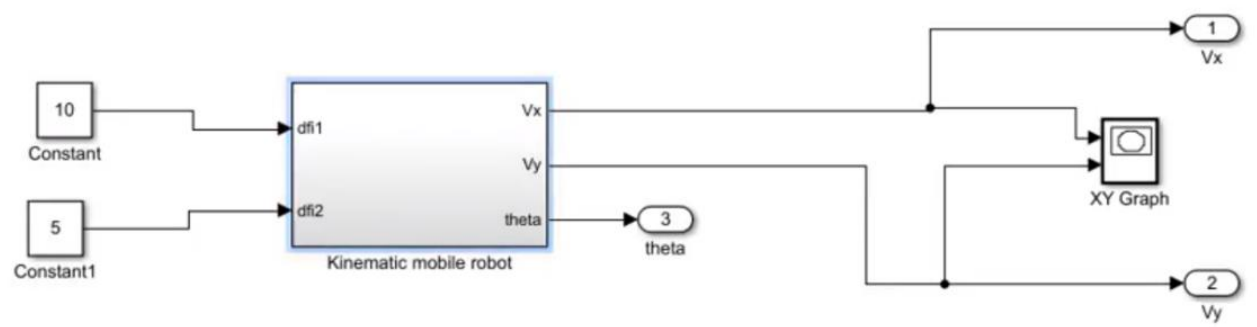

Fig. 5. Graphical-numerical model of a wheeled robot in the environment of Matlab/Simulink. 


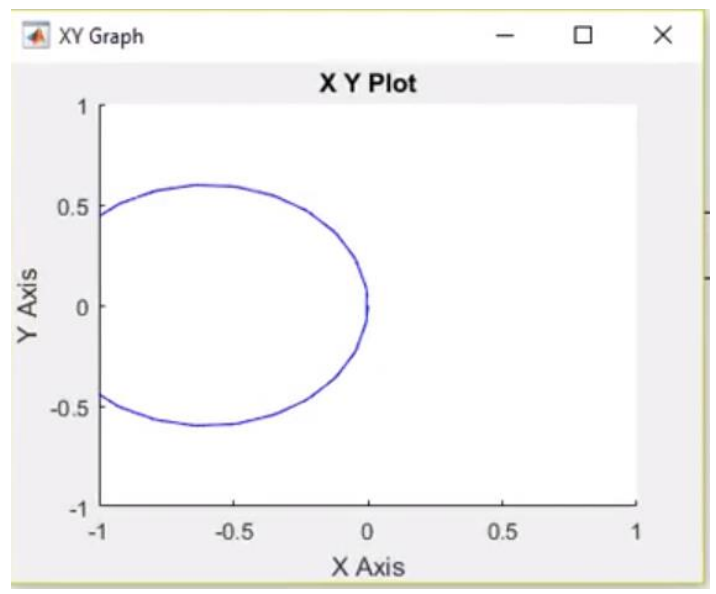

Fig. 6. The mass center trajectory of the wheeled robot model.

\section{Conclusions}

Based on the results of mathematical modeling of the wheeled robot's (with two independent wheels located on the same axis motion along a plane), the robot was simulated in the Matlab/Simulink programming environment. The voltage applied to the wheel drive Constant and Constantl was differed by a factor of two, which allowed obtaining the mass center trajectory of the robot at the point $O_{0}$ in the form of a circle, thereby confirming the correctness of mathematical modeling.

The developed mathematical model for controlling the movement of a wheeled robot will allow providing the efficient interaction between the electric drive system, positioning and the central control board that regulates the number of impulses given to the wheeled robot's drive with digital autonomous motion control.

The research was carried out as part of the State Program for the Development of Agriculture for 2013-2020 and Decree of the Governmant of the Russian Federation of August 25, 2017 No. 996 "Ob utverzhdenii nauchno-tekhnicheskoi programmy razvitiia selskogo khoziaistva na 2017-2020"

\section{References}

1. E.A. Nikitin, A.S. Dorokhov, D.Yu. Pavkin, Machinery and equipment for the village 33, 32-34 (2019). DOI: 10.33267/2072-9642-2019-11-32-34/j.

2. Parray, Rouf, Mani, Indra, Kumar, Adarsh, Khura, Tapan, Lande, Satish, Indian Journal of Agricultural Sciences 86, 167-172 (2016)

3. S.N. Subhashree, C. Igathinathane, Biosystems engineering 187, 185-200 (2019). DOI: 10.1016/j.biosystemseng.2019.08.018

4. D.A. Crolla, A.S.A. El-Razaz, Journal of Terramechanics 24(3), 199-225 (1987). DOI:10.1016/0022-4898(87)90041-3

5. S. Mileiko, T. Bunnam, F. Xia, R. Shafik, A. Yakovlev, S. Das, Philosophical Transactions of the Royal Society A Mathematical, Physical and Engineering Sciences 378(2164), 20190166 (2019). DOI:10.1098/rsta.2019.0166

6. Q. Chen, C. Liao, A. Ouyang, X. Li, Q. Xiao, International Journal of Electric and Hybrid Vehicles 8(3), 242 (2016). DOI:10.1504/ijehv.2016.080024 
7. J. Mahalakshmi, K. Kuppusamy, C. Kaleeswari, P. Maheswari, Emerging Technologies for Agriculture and Environment, 39-52 (2019). DOI:10.1007/978-98113-7968-0_4

8. P. He, J. Li, Applied Soft Computing, (2019). DOI:10.1016/j.asoc.2019.01.040

9. A. Tahouni, M. Mirzaei, B. Najjari, Journal of Automobile Engineering, 095440701984885 (2019). DOI:10.1177/0954407019848858

10. O.C. Barawid, A. Mizushima, K. Ishii, N. Noguchi, Biosystems Engineering 96(2), 139-149 (2007). DOI:10.1016/j.biosystemseng.2006.10.012 